\title{
A entrevista motivacional em tabagistas: uma revisão teórica*
}

\author{
Motivational interview in smokers: a theoretical review
}

\author{
Wilson Vieira Melo'; Margareth da Silva Oliveira'I; Renata Brasil Araújo'l'; Rosemeri \\ Siqueira Pedroso'v
}

\begin{abstract}
'Psicólogo. Doutorando em Psicologia, Universidade Federal do Rio Grande do Sul (UFRGS), Porto Alegre, RS. Diretor, WP Centro de Psicoterapia Cognitivo-Comportamental, Porto Alegre, RS. Professor, Curso de Psicologia, Faculdades Integradas de Taquara (FACCAT), Taquara, RS. Professor, Curso de Especialização em Terapia Cognitivo-Comportamental, WP Centro de Psicoterapia Cognitivo-Comportamental, Porto Alegre, RS.

"Psicóloga. Doutora em Ciências, Universidade Federal de São Paulo (UNIFESP), São Paulo, SP. Professora, Cursos de Graduação e Pós-Graduação, Faculdade de Psicologia, Pontifícia Universidade Católica do Rio Grande do Sul (PUCRS), Porto Alegre, RS. Coordenadora, Grupo de Pesquisa Intervenções Cognitivas e Comportamentos Dependentes, PUCRS, Porto Alegre, RS.

IIIPsicóloga. Doutora em Psicologia, PUCRS, Porto Alegre, RS. Especialista em Dependência Química pela Cruz Vermelha. Coordenadora, Residência Integrada e Unidade de Dependência Química, Hospital Psiquiátrico São Pedro, Porto Alegre, RS.

IVPsicóloga. Mestre em Psicologia Clínica, PUCRS, Porto Alegre, RS.
\end{abstract}

Endereço para correspondência

Rev Psiquiatr RS. 2008; 30(1 Supl).

\section{RESUMO}

O tabagismo é uma das principais causas de morte prematura em todo o mundo, além de ser uma das dependências de mais difícil tratamento. Uma das abordagens que vêm sendo utilizadas no tratamento da dependência do tabaco é a entrevista motivaciona, baseada no modelo transteórico proposto por Prochaska \& Di Clemente. Este trabalho tem por objetivo realizar uma revisão teórica a respeito dos estágios motivacionais de mudança e do uso da entrevista motivacional breve em pacientes tabagistas. Apresentam-se, no decorrer do texto, resultados de algumas das mais importantes pesquisas realizadas na Europa e nos Estados Unidos acerca do assunto. Os resultados indicam que, apesar de a entrevista motivacional breve apresentar bons resultados no tratamento de comportamentos aditivos, quando comparada a outras modalidades de intervenção, esse sucesso não parece se estender aos 
resultados encontrados no tratamento do tabagismo.

Descritores: Entrevista motivacional, tratamento, tabagismo, estágios motivacionais.

\section{ABSTRACT}

Smoking is one of the main causes of premature death worldwide. It is also an addiction that requires a difficult treatment. One approach that has been used to treat tobacco addiction is motivational interview, based on the transtheorical model proposed by Prochaska \& Di

Clemente. This study aims at performing a theoretical review concerning motivational stages of change and the use of brief motivational interview in smokers. The results obtained from some of the most important studies on the subject conducted in Europe and in the USA are presented here. These results indicate that, although brief motivational interview shows good outcomes concerning the treatment of addictive behaviors when compared with other categories of intervention, such success does not seem to apply to the results found in the treatment of smoking.

Keywords: Motivational interview, treatment, smoking, motivational stages.

\section{NTRODUÇÃO}

O tabagismo é um comportamento que foi socialmente tolerado e até exaltado durante muito tempo. Hoje em dia, o tabaco é um problema de saúde pública que atinge milhares de pessoas em todo o mundo ${ }^{1}$.

Dentre as causas de morte prematura evitáveis em todo o mundo, podemos citar o tabaco como certamente sendo uma das principais. De acordo com a Organização Mundial da Saúde², 4 milhões de pessoas morrem a cada ano devido a doenças causadas diretamente pelos derivados do cigarro, o que corresponde, em média, a 10 mil mortes a cada dia. Caso mantenha-se a tendência atual, no ano 2025 já serão 10 milhões de mortes anuais causadas pelo tabaco. A menos que se faça alguma coisa a esse respeito, do 1,1 bilhão de fumantes, 500 milhões morrerão por causa dos cigarros ao longo das próximas décadas. Porém, muitas dessas mortes poderiam ser potencialmente evitáveis se essas pessoas pararem de fumar ${ }^{1}$.

Indubitavelmente, um dos principais obstáculos encontrados na tentativa de interromper o uso do tabaco é a motivação. Heather ${ }^{3}$ resumiu isso quando comentou que "os transtornos aditivos são essencialmente problemas motivacionais". Durante muito tempo, acreditou-se que a resistência era o resultado de traços de personalidade nocivos e que os pacientes são em si mesmos desmotivados para a mudança, principalmente quando falamos de comportamentos aditivos. Contudo, crê-se atualmente que a motivação não deve ser pensada como um problema de personalidade, e sim como um estado de prontidão ou de avidez para a mudança, que pode oscilar de tempos em tempos ou de uma situação para outra ${ }^{4}$.

A entrevista motivacional, conforme proposta por Miller $\&$ Rollnick ${ }^{4}$, é uma alternativa viável no tratamento de comportamentos aditivos, dentro das intervenções breves, uma vez que o impacto inicial parece ter influência na motivação para a mudança de comportamento 4,5 . Segundo Miller \& Rollnick ${ }^{4}$, tudo indica que o aconselhamento relativamente breve pode ter um impacto substancial, uma vez que aqueles que recebem um aconselhamento breve tecnicamente bem planejado mostram muito mais melhoras do que os que não recebem nenhuma orientação. 


\section{OBJ ETI VO}

O presente trabalho tem como objetivo descrever uma revisão teórica a respeito do uso da entrevista motivacional, de acordo com Miller $\&$ Rollnick ${ }^{4}$, em pacientes tabagistas, enfatizando aspectos relacionados à intervenção breve e à transição entre os estágios motivacionais propostos pelo modelo transteórico, descrito pelos psicólogos James Prochaska \& Carlo Di Clemente ${ }^{6}$.

\section{MÉTODO}

Foi realizada uma pesquisa no banco de dados Psychlnfo, de artigos indexados, publicados entre os anos de 1999 e 2004, além de alguns artigos importantes citados nessas publicações. Foram incluídos todos os artigos encontrados a partir da utilização dos descritores motivational interview, tobacco, tobaccoism and motivation. Ademais, foram pesquisados livros de autores importantes da construção da teoria ${ }^{1,4,5,7-11}$, bem como uma dissertação de mestrado ${ }^{12}$ acerca do assunto, escrita no Brasil.

\section{Tabagismo}

Atualmente, pode-se dizer que a maioria das pessoas sabe que o cigarro traz malefícios à saúde, mas muitas delas não sabem as proporções desses prejuízos. Além do câncer - pois cerca de $30 \%$ de todos os casos são causados pelo tabaco -, outras doenças graves estão intimamente ligadas ao uso do cigarro, tais como enfisema pulmonar, bronquite e doenças cardiovasculares. Além disso, úlceras do estômago e do duodeno, osteoporose, infecções respiratórias - inclusive pneumonias - e diversos problemas dentários podem ter como origem ou agravamento o uso do cigarro. O tabagismo também afeta o desenvolvimento da gravidez, uma vez que filhos de mães fumantes nascem com cerca de 200 gramas a menos do que quando comparados com outros bebês e estão sujeitos a apresentar morte súbita e outras doenças peri e neonatais ${ }^{1}$.

A cadeia epidemiológica é bastante extensa, indo desde a agregação de fatores de risco e a comorbidade freqüente no domínio da psicopatologia até as intoxicações por agrotóxico nas áreas de produção e manipulação do produto bruto e manufaturado, sem contar as relações obscuras com o tráfico e a comercialização de outras substâncias aditivas. Economicamente, o tabagismo leva a altos custos sociais, tanto em gastos com a saúde quanto em perda da produtividade e morte precoce ${ }^{7}$. O custo social do tabagismo nos Estados Unidos foi estimado em mais de 100 bilhões de dólares, devido à perda de produtividade e à morbimortalidade ${ }^{13}$.

Os efeitos farmacológicos da nicotina são diversos e dependem do número de cigarros e da freqüência do fumo, bem como do desenvolvimento da tolerância. A nicotina promove um rápido estado de alerta, melhorando a atenção, a concentração e a memória, semelhante àquele descrito pelos usuários de cocaína. Esse efeito, associado aos sintomas desagradáveis da falta da substância no cérebro, contribui para a dificuldade de alcançar a manutenção da abstinência dessa droga, pois, entre os fumantes que já tentaram parar de usar tabaco, cinco a sete tentativas são necessárias até se atingir o objetivo ${ }^{7,14}$. 
De um modo geral, é possível se dizer que o início do consumo de tabaco se dá na adolescência, por volta dos 13 aos 15 anos em $90 \%$ dos casos. Os fatores que motivam tantos jovens a experimentar o cigarro são socioculturais. Fatores individuais que podem contribuir para o início do consumo de cigarros ainda são pouco conhecidos, mas incluem fatores hereditários (genéticos), eventualmente relacionados à modulação dos efeitos da nicotina e do humor ${ }^{1}$.

Quando a dependência da nicotina já se encontra instalada, a manutenção do comportamento de fumar começa a ser gradativa e fortemente associada a causas psicológicas, como condicionamentos e crenças acerca da substância e do uso, além das bases neuroquímicas e genéticas envolvidas ${ }^{15}$.

\section{Estágios motivacionais}

O modelo transteórico de Prochaska \& Di Clemente ${ }^{6}$ descreve a prontidão para a mudança como estágios nos quais o indivíduo transita. Os autores destacam cinco estágios bem definidos, confiáveis e relacionados entre si: pré-contemplação, contemplação, preparação, ação e manutenção.

A pré-contemplação é um estágio em que não há intenção de mudança, pela falta de consciência dos prejuízos associados ao comportamento-problema; a contemplação se dá quando há uma crítica quanto à existência do problema, mas ainda não foi iniciado nenhum tipo de ação, devido à ambivalência; a preparação ocorre quando há uma decisão de mudar, porém ainda nenhuma ação é empreendida; a ação é o estágio no qual o cliente toma uma atitude; e a manutenção é quando se trabalha a prevenção à recaída e a consolidação dos ganhos obtidos durante a ação 4,6 .

A utilidade do modelo dos estágios motivacionais, de Prochaska \& Di Clemente ${ }^{6}$, tem sido demonstrada no campo dos comportamentos aditivos em um grande número de publicações nos últimos anos ${ }^{16}$.

Da "pré-contemplação", através da "contemplação" e a "ação", para a "manutenção", o modelo compreende o movimento através dos estágios, freqüentemente envolvendo uma reincidência no processo de recaída. Em uma importante revisão sobre o modelo, a recaída não é vista como um estágio, mas como um evento que termina o estágio de "ação" ou de "manutenção" e pontua um movimento de volta aos estágios iniciais de "pré-contemplação" e "contemplação"17. O que torna esse modelo tão atraente é que se propõem diferentes intervenções e estratégias de tratamento para os diferentes estágios de mudança ${ }^{8-10,18}$.

Porém, o conceito de estágios tem sofrido algumas críticas por parte de autores importantes. De acordo com Sutton ${ }^{11}$, o conceito de estágio implica ordenação e seqüência, uma vez que supõe que alguém, num determinado estágio, teria vindo do estágio precedente na seqüência e esteja a caminho do próximo estágio. O que esse autor sugere é que se utilize o termo "estado de mudança" ao invés de "estágio de mudança", uma vez que essa organização linear, muitas vezes, não é observada na prática. Os modelos mais recentes adaptaram a roda da mudança, como é conhecida, a um formato de espiral, na tentativa de dar conta do dinamismo que envolve os processos motivacionais, já que existe uma evolução na motivação do indivíduo cada vez que ele passa pelos estágios.

Em um destacado estudo de seguimento, foram relatados dados de uma amostra de 544 sujeitos que se automodificaram. Os participantes forneceram informações sobre o estágio motivacional a cada 6 meses por um período de 2 anos. Os padrões de movimento através dos estágios não são relatados completamente, mas é possível extrair alguns achados importantes do artigo. Em especial, tem-se que apenas $16 \%$ dos sujeitos apresentaram uma progressão estável, no decorrer dos 2 anos, de um estágio para o seguinte na seqüência. Além disso, aparentemente, não houve nenhum sujeito apresentando uma progressão estável através de três ou mais estágios, uma vez que o máximo foi em dois estágios. Outro dado significativo foi 
que $12 \%$ dos sujeitos retrocederam um ou dois estágios, e $36 \%$ apresentaram um perfil plano, ou seja, ficaram predominantemente no mesmo estágio nos cinco momentos de mensuração ${ }^{19}$. Dessa forma, os achados relatados mostram que o movimento progressivo para frente através dos estágios está longe de ser o padrão habitual de mudança entre os voluntários que se automodificam ${ }^{11,20}$.

Ainda de acordo com Sutton ${ }^{11}$, uma outra limitação desse modelo encontra-se no fato de que o mesmo não diz quanto tempo as pessoas ficam num determinado estágio ou quando e sob quais circunstâncias elas mudam sua situação (exceto no caso do movimento entre "ação" e "manutenção"). Portanto, para esse autor, o modelo dos estágios de mudança não é um modelo que descreve quando as pessoas mudam, como por vezes se afirma.

A crítica de Sutton também é passível de ser questionada, uma vez que, dado o dinamismo das condições motivacionais, 6 meses é tempo suficiente para que o paciente percorra a roda da mudança, mais do que uma vez inclusive. Além disso, o modelo demonstra ser bom no que diz respeito ao seu caráter explicativo, porém com limitações claras quanto ao diagnóstico e predição de mudança.

Instrumentos de avaliação

Têm-se tentado operacionalizar e mensurar os estágios de mudança com o uso de alguns instrumentos de medida. Uma das principais alternativas para a definição categórica de estágios é a escala University of Rhode I sland Change Assessment (URICA). Esta consiste de 32 itens, oito para cada um dos quatro estágios (o estágio de preparação não está apresentado). Para estudar a confiabilidade e validade, a escala foi administrada em duas amostras clínicas separadas $^{21,22}$. Em ambos os casos, a análise fatorial (componentes principais) apresentou um resultado semelhante de quatro fatores.

Além da escala URICA, outros instrumentos de avaliação também têm sido estudados na tentativa de se avaliar objetivamente os estágios motivacionais de mudança ${ }^{23}$. Dentre os principais instrumentos, destacam-se: o questionário Readiness to Change Questionnaire $(\text { RTCQ })^{24}$; a escala Stages of Change Readiness and Treatment Eagerness Scale (SOCRATES) ${ }^{25}$; a escala Texas Christian University Motivational Assessment Scale (TCU) ${ }^{26}$; a escala Circumstances, Motivation, Readinessand Sultability Scale (CMRS) ${ }^{27}$; o questionário Recovery Attitude and Treatment Evaluator (RAATE) ${ }^{28}$; o questionário Treatment Motivation Questionnaire (TMQ) ${ }^{29}$; e a escala Substance Abuse Treatment Scale (SATS) ${ }^{30}$.

A entrevista motivacional: intervenção breve

A entrevista motivacional é uma abordagem terapêutica que utiliza um modo particular de ajudar as pessoas a reconhecerem e fazerem algo a respeito de seus problemas presentes ou potenciais ${ }^{4}$ e que se fundamenta no modelo transteórico de Prochaska \& Di Clemente ${ }^{6}$. É uma importante ferramenta utilizada para trabalhar a ambivalência, presente na maioria dos comportamentos aditivos, de uma forma mais persuasiva do que coerciva.

Essas técnicas podem ser trabalhadas em um número reduzido de sessões, podendo, inclusive, resumir-se a um único encontro. Contudo, a intervenção breve tem sido realizada em um número que gira de um a três encontros ${ }^{4}$. Os elementos que norteiam a intervenção breve parecem ser os "princípios ativos" desse modelo de intervenção e foram reunidos no acróstico FRAMES, ou seja, F: feedback, R: responsibility, A: advice, M: menu, E: empathy e S: selfefficacy. No Brasil, durante o X Congresso Brasileiro de Alcoolismo e Outras Dependências, em um curso ministrado pelo Dr. Willian Miller, o acróstico foi apresentado e transposto para o português como ADERIR, sendo A: auto-eficácia (self-efficacy), D: devolução (feedback), E: empatia (empathy), R: responsabilidade (responsibility), I: inventário (menu) e R: recomendações (advice). 
Diversos estudos têm sido realizados na tentativa de demonstrar a eficácia da entrevista motivacional em tabagistas ${ }^{31-35}$.

Um estudo de meta-análise avaliou resultados de ensaios clínicos controlados investigando adaptações da entrevista motivacional em diversas áreas de comportamentos-problema ${ }^{16}$. Os critérios de inclusão dos artigos eram: serem relacionados a intervenções que utilizassem os princípios da entrevista motivacional ${ }^{6}$, que a estas fossem individuais e que fossem ensaios clínicos randomizados, com pelo menos um grupo controle. Os resultados encontrados pelos pesquisadores foram equivalentes a outras formas de tratamento e apresentaram efeitos moderados (de 0,25 a 0,57 ), quando comparados a grupos sem nenhum tratamento e/ ou placebo para problemas como alcoolismo, uso de drogas ilícitas, problemas com dietas e exercícios físicos. Os resultados da meta-análise, apesar de demonstrarem a eficácia da entrevista motivacional para diversos comportamentos aditivos, não sustentaram sua eficácia para comportamentos de risco para HIV/AIDS e nem para o tratamento do tabagismo ${ }^{16}$. 0 tamanho de efeito encontrado para o tratamento envolvendo a entrevista motivacional em tabagistas, quando comparado a placebo ou grupos sem tratamento, foi de $0,11(-0,05$ a 0,27$)$. Contudo, os resultados encontrados nessa meta-análise podem ter-se dado devido ao número de estudos envolvidos na revisão. Apenas duas pesquisas foram avaliadas e incluídas no estudo de meta-análise, dada a rigorosidade dos critérios de inclusão na seleção dos estudos ${ }^{33,34}$.

Em mais outro importante estudo realizado, foi avaliada a eficácia de três grupos de tratamento para o tabagismo, sendo eles: 1 ) cognitivo-comportamental (treinamento de habilidades); 2 ) entrevista motivacional; e 3) intervenção breve, que não a entrevista motivacional, em uma amostra randomizada de 677 pacientes $^{35}$. A diferença entre os três grupos de tratamentos também foi avaliada em dois subgrupos classificados em alto e baixo risco de recaída, levandose em consideração escores de dependência de nicotina, níveis de intoxicação por monóxido de carbono, sintomas de ansiedade e de depressão, número de cigarros diários, anos de fumo, idade, gênero, dentre outras variáveis. A intervenção breve consistia de três sessões no formato individual, e a terapia cognitivo-comportamental e a entrevista motivacional, de seis sessões em grupo. Os participantes dos dois primeiros grupos também receberam a intervenção breve antes do tratamento grupal dispensado. Além disso, todos os participantes receberam 8 semanas de tratamento com adesivos de reposição de nicotina. Os resultados indicaram que, surpreendentemente, não houve diferença entre nenhum dos três grupos de tratamento oferecidos, nem nos fumantes com alto risco de recaída, nem nos que foram considerados com baixos fatores de risco para recaída ${ }^{35}$.

Em um estudo realizado com 365 adolescentes americanos com idade entre 14 e 19 anos, média de 16,21 (desvio padrão $=1,11$ ), foi comparada uma intervenção breve da entrevista motivacional ( $n=180$ ) com um programa escolar de intervenção curricular $(n=185)$. Os critérios de inclusão consistiam na exigência de que os adolescentes precisavam estar fumando cinco cigarros diários ou mais. Os resultados demonstraram que a intervenção breve da entrevista motivacional teve sucesso apenas com fumantes leves, e o programa escolar de intervenção curricular foi mais efetivo com fumantes com um índice maior de dependência de nicotina, incluindo os dependentes graves ${ }^{36}$.

Outro estudo realizado com uma amostra randomizada de 200 adultos jovens ingleses, com idades entre 16 e 20 anos, comparou a eficácia da entrevista motivacional ( $n=105$ ) com nenhuma intervenção além do controle usual realizado pela escola. Os participantes eram usuários de maconha, álcool e outros estimulantes não-injetáveis, incluindo a nicotina. A entrevista motivacional foi realizada em uma única sessão individual, estruturada em tópicos com duração de 1 hora. Após 3 meses, os adultos jovens foram reavaliados, e os resultados apontados foram de que a entrevista motivacional reduziu o uso de cigarro, álcool e maconha. O tamanho de efeito para a redução do consumo de tabaco foi de $0,37(0,15-0,6)^{37}$. 
que se encontravam entre a $28^{a}$ e a $34^{a}$ semana de gestação, e também na sexta semana, no terceiro e no sexto mês de pós-parto, todas as pacientes receberam um aconselhamento de 3 a 5 minutos, mais um folheto informativo na sua primeira consulta pré-natal e sete folhetos informativos semanalmente enviados para as suas residências via correio. O grupo experimental recebeu uma carta com um feedback personalizado de seu estágio motivacional de mudança e dois telefonemas de aconselhamento usando estratégias da entrevista motivacional. O grupo controle, por sua vez, recebeu os cuidados usualmente empregados pelo serviço. Os dados de coleta de cotinina na $34^{a}$ semana demonstraram que não houve diferença entre os grupos de casos e controles. Entretanto, o grupo de 6 semanas de pós-parto demonstrou que $43 \%$ das pacientes que receberam a intervenção motivacional pararam de fumar, comparado com 34\% do grupo controle. Os grupos pós-parto de 3 e 6 meses também não apresentaram diferença estatisticamente significativa. Os dados sugerem resultados modestos da entrevista motivacional com gestantes resistentes ao tratamento do tabagismo ${ }^{38}$.

Ainda no que se refere ao tratamento do tabagismo com mulheres grávidas, outro importante estudo merece destaque. Nele, foi avaliada uma amostra de 390 mulheres fumantes com mais de 18 anos. As participantes foram randomizadas em três grupos distintos de tratamento: 1) um livreto de auto-ajuda especialmente construído para gestantes fumantes; 2) o livreto mais acesso a um programa de apoio via telefone, onde a gestante interagia com um programa computadorizado de voz; e 3) o livreto mais aconselhamento telefônico realizado por enfermeiras, utilizando as técnicas e estratégias da entrevistas motivacional. Nenhuma tentativa adicional de auxílio ao tratamento do tabagismo foi realizada no acompanhamento pré-natal. As medidas de cotinina coletadas através da urina na 34a semana de gravidez confirmavam bioquimicamente a abstinência ou não. Não houve diferença estatisticamente significativa entre os três grupos, e apenas $20 \%$ das pacientes conseguiram se manter abstinentes após as intervenções ${ }^{39}$.

De acordo com Rodriguez ${ }^{12}$, em sua dissertação de mestrado defendida na Pontifícia Universidade Católica do Rio Grande do Sul, intitulada "Intervenção Motivacional Breve para Tabagismo Aplicada a Alcoolistas Fumantes", foram estudados 38 pacientes alcoolistas fumantes internados para o tratamento do alcoolismo. Foi aplicada a entrevista motivacional em uma única sessão em grupo. Os resultados foram os de que, após a intervenção motivacional breve direcionada para o tabagismo, não houve modificações estatisticamente significativas na motivação para parar de fumar tabaco, apesar de a maioria dos sujeitos ter sido classificada como tendo um grau de dependência leve a moderado da substância. Os resultados, porém, podem estar enviesados pelo tamanho reduzido da amostra, pela internação dos pacientes no momento da intervenção, além de possuírem níveis graves de alcoolismo, o que sabidamente é um fator de dificuldade para o tratamento do tabagismo.

\section{DISCUSSÃO}

O tabagismo é uma das dependências mais difíceis de se tratar, e são necessárias, em média, de cinco a sete tentativas prévias para que se consiga atingir a abstinência ${ }^{7,13}$. Um dos fatores que contribuem para essa estatística é a motivação para parar de fumar, ou seja, a prontidão para a mudança.

A entrevista motivacional é uma intervenção que reúne muito mais do que um conjunto de técnicas para fazer-se um aconselhamento, uma vez que ela é uma maneira de estar-se com o paciente, provavelmente de um modo bastante diferente da forma como os outros podem tê-lo tratado no passado ${ }^{4}$. É uma forma de tratamento bastante promissora e que dá alento ao difícil tratamento de comportamentos aditivos. 
Diversos estudos têm sido realizados na tentativa de se comprovar a efetividade da entrevista motivacional breve no tratamento do tabagismo; entretanto, os resultados não são tão satisfatórios, principalmente quando se fala de pacientes com dependência grave de tabaco ${ }^{31-}$ 35. Certamente, é de fundamental importância a realização de mais estudos com pacientes tabagistas graves, principalmente com o uso de técnicas da entrevista motivacional com um maior número de sessões, dado o grande potencial dependógeno da nicotina.

Outra razão para esses modestos resultados em relação ao desempenho da entrevista motivacional breve, no que se refere ao tratamento do tabagismo ${ }^{31-35}$, pode ser oriunda do fato de que pesquisas relacionadas à psicoterapia de um modo geral, seja ela em grupo ou individual, são de difícil realização. Essa dificuldade em se operacionalizar esse tipo de estudo deve-se ao grande número de variáveis envolvidas, tais como nível do treinamento das equipes e garantia da homogeneização do uso das técnicas. Em suma, é difícil ter certeza com relação aos métodos empregados nos tratamentos psicoterápicos.

Desta sorte, não se pode utilizar esse tipo de argumentação para justificar possíveis limitações nos resultados das pesquisas no que tange ao uso da entrevista motivacional no tratamento do tabagismo. Os resultados da literatura claramente demonstram boa resposta terapêutica no tratamento dos demais comportamentos aditivos ${ }^{16}$, o que não se repete no caso do tabagismo, apesar de a argumentação anterior quanto ao método dos estudos também ser válida para os demais estudos envolvendo outras dependências.

Outro fator importante a ser destacado é que os instrumentos para avaliação dos estágios motivacionais ${ }^{21-30}$, desenvolvidos com base nos princípios que norteiam e fundamentam a teoria do modelo transteórico ${ }^{6}$, propõem-se a avaliar em qual dos estágios o funcionamento do sujeito está predominantemente concentrado. Apesar disso, alguns desses instrumentos $21,22,25$ não possuem subescalas capazes de avaliar o estágio de preparação, por exemplo, uma vez que esse estágio é de difícil definição, muitas vezes sendo confuso o estabelecimento das fronteiras com os demais estágios.

Outro ponto a ser destacado é que alguns desses instrumentos, como é o caso das escalas URICA $^{21,22}$ e SOCRATES ${ }^{25}$, funcionam, na verdade, como várias escalas contidas em uma só e não como subescalas que têm uma lógica clínica e podem ser analisadas em conjunto. Um indivíduo dependente poderá ter, por exemplo, muitos pontos no estágio contemplação, ainda que esteja "convictamente" na manutenção, com um longo tempo em abstinência e com uma forte crítica a respeito do significado que uma recaída teria em sua vida.

É preciso salientar que o presente artigo não foi uma revisão sistemática, não tendo sido feito um contato com os autores das pesquisas analisadas para investigar a existência de artigos ainda não publicados a respeito da entrevista motivacional com tabagistas. Essa limitação pode ter impedido de incluir, nesta revisão, ensaios clínicos mais recentes que utilizassem essa intervenção com, por exemplo, um número maior de sessões, o que poderia, talvez, melhorar a sua efetividade no caso dos tabagistas mais graves.

\section{CONCLUSÕES}

O tabagismo é, indubitavelmente, um dos comportamentos aditivos mais difíceis de se tratar, dada a quantidade de fatores que estão envolvidos na gênese, desenvolvimento e manutenção da sua dependência, que incluem desde condicionamentos e estados de humor até fatores genéticos e sociais. 
Apesar de a entrevista motivacional breve ser uma das formas de tratamento que mais tem demonstrado resultados no campo dos comportamentos aditivos nos últimos anos, esses resultados não são tão satisfatórios quando falamos de dependentes - principalmente os graves - do tabaco ${ }^{15}$.

No entanto, verifica-se que ainda não existem pesquisas capazes de avaliar com segurança a eficácia/efetividade da entrevista motivacional breve nos tabagistas, já que a metodologia e as amostras utilizadas são muito diversas, sendo difícil uma comparação e uma generalização de seus resultados.

O fato de a entrevista motivacional breve não parecer funcionar para tabagistas graves não significa que essa abordagem, com um número ampliado de sessões ou associada a outras terapêuticas - como tratamento farmacológico combinado -, não possa ser mais efetiva no tratamento dessa clientela. Assim, é necessário que sejam realizadas novas pesquisas para avaliar a entrevista motivacional breve em populações de tabagistas, pois seus resultados podem tornar possível a instrumentalização dos terapeutas para o aumento das taxas de sucesso em suas práticas clínicas.

\section{REFERÊNCI AS}

1. Gigliotti A, Carneiro E, Ferreira M. Tratamento do tabagismo. In: Range B. Psicoterapias cognitivo-comportamentais: um diálogo com a psiquiatria. Porto Alegre: Artmed; 2001. p. 35171.

2. World Health Organization (WHO). Confronting the tobacco epidemic in an era trade liberation. Geneva: WHO; 2003.

3. Heather N. Addictive disorders are essentially motivational problems. Br J Addict. 1992;87

(6): 828-30.

4. Miller WR, Rollnick S. Entrevista motivacional: preparando as pessoas para a mudança de comportamentos adictivos. Porto Alegre: Artmed; 2001.

5. Miller WR, Brown JM. Self-regulation as a conceptual basis for the prevention and treatment of addictive behaviors. In: Heather N, Miller WR, Greeley J editors. Self-control and addictive behaviors. Sydney: Pergamon; 1991. p. 3-79.

6. Prochaska JO, DiClemente CC. Transtheoretical therapy toward a more integrative model of change. Psychother Theory Res Pract. 1982: 19(3):276-87.

7. Achutti A, coordenador. Guia nacional de prevenção e tratamento do tabagismo. Rio de J aneiro: Vitrô Comunicação \& Editora; 2001.

8. Davidson R, Rollnick S, MacEwan I. Counseling problem drinkers. London:

Tavistock/Routledge; 1991.

9. DiClemente CC, Prochaska JO. Processes and stages of change of self-change: coping and competence in smoking behavior change. In: Shiffman S, Wills TA. Coping and substance abuse. New York: Academic; 1985. p. 319-43. 
10. Prochaska JO, Norcross JC, DiClemente CC. Changing for good: a revolutionary six-stage program for overcoming bad habits and moving your life positively forward. New York: Quill; 1994.

11. Sutton S. Os Estágios de mudança podem orientar o tratamento das adições? Um exame crítico do modelo de Prochaska e DiClemente. In: Edwards G, Dare C, organizador. Psicoterapia e tratamento de adições. Porto Alegre: Artmed; 1997. p. 173-88.

12. Rodriguez VC. Intervenção motivacional breve para tabagismo aplicada a alcoolistas fumante [dissertação]. Porto Alegre: Pontifícia Universidade Católica do Rio Grande do Sul; 2003.

13. Ramsay J, Hoffmann A. Smoking cessation and relapse prevention among undergraduate students: a pilot demonstration project. J Am Coll Health. 2004;53(1):11-8.

14. Gigliotti A, Bonetto DVS, Alves JG, Jardim JRB, Maranhão MFC, Zamboni M, et al. Tabagismo. J Bras Med. 1999; 77(2): 48-78.

15. O'Connell M, Lucan SC, Yeh MC, Rodriguez E, Shah D, Chan W, et al. Impediment profiling for smoking cessation: results of a pilot study. Am J Health Promot. 2003; 17(5):300-3.

16. Burke BL, Arkowitz $\mathrm{H}$, Menchola $\mathrm{M}$. The efficacy of motivational interviewing: a metaanalysis of controlled clinical trials. J Consult Clin Psychol. 2003; 71(5):843-61.

17. DiClemente CC, Prochaska JO, Fairhurst SK, Velicer WF, Velasquez MM, Rossi JS. The process of smoking cessation: an analysis of the precontemplation, contemplation and preparation stages of change. J Consult Clin Psychol. 1991;59(2):295-304.

18. Prochaska J O, Velicer WF, DiClemente CC, Fava J. Measuring processes of change: applications to the cessation of smoking. J Consult Clin Psychol. 1988;56(4):520-8.

19. Prochaska JO, Velicer W, Guadagnoli E, Rossi JS, DiClemente CC. Patterns of change: dynamic typology applied to smoking cessation. Multivariate Behav Res. 1991;26:83-107.

20. Etter JF, Sutton S. Assessing "stage of change" in current and former smokers. Addiction. 2002; 97(9): 1171-82.

21. McConnaughy EA, Prochaska JO, Velicer WF. Stages of change in psychoterapy: measurement and samples profiles. Psychoter: Theory, Res Pract. 1983; 20:368-75.

22. McConnaughy EA, DiClemente CC, Prochaska J O, Velicer WF. Stages of change in psychotherapy: a follow-up report. Psychother: Theory, Res Pract. 1989; 26: 494-503.

23. Carey KB, Purnine DM, Maisto SA, Carey MP. Assessing readiness to change substance abuse: A critical review of instruments. Clin Psychol. 1999;6(3):245-66.

24. Budd R, Rollnick $S$. The structure of the readiness to change questionnaire: a test of Prochaska \& DiClemente's transtheoretical model. Br J Health Psychol. 1996; 1:365-76.

25. Miller WR, Tonigan JS. Assessing drinkers' motivation for change: The Stages of Change Readiness and Treatment Eagerness scale (SOCRATES). Psychol Addict Behav. 1996; 10(2):819.

26. Simpson DD, Chatham LR. TCU/DATAR forms manual: Improving Drug Abuse Treatment, Assessment, and Research (DATAR) project. Fort Worth, TX: Texas Christian University, Institute of Behavioral Research. 1995. 
27. De Leon G, Melnick G, Kressel D, Jainchill N. Circumstances, motivation, readiness, and suitability (the CMRS scales): Predicting retention in therapeutic community treatment. Am J Drug Alcohol Abuse. 1994;20(4):495-515.

28. Mee-Lee $D$. An instrument for treatment progress and matching: the recovery attitude and treatment evaluator (RAATE). J Subst Abuse Treat. 1988;5(3): 183-6.

29. Ryan RM, Plant RW, O'Malley S. I nitial motivations for alcohol treatment: Relations with patient characteristics, treatment involvement, and dropout. Addict Behav. 1995;20(3):279-97.

30. McHugo GJ, Drake RE, Burton HL, Ackerson TH. A scale for assessing the stage of substance abuse treatment in persons with severe mental illness. J Nerv Ment Dis. 1995; 183(12): 762-7.

31. Hughes JR. Motivating and helping smokers to stop smoking. J Gen Intern Med. 2003;18 (12): 1053-7.

32. Emmons KM, Rollnick S. Motivational interviewing in health care settings. Opportunities and limitations. Am J Prev Med. 2001;20(1):68-74.

33. Colby SM, Monti PM, Barnett NP, Rohsenow DJ, Weissman K, Spirito A, et al. Brief motivational interviewing in a hospital setting for adolescent smoking: A preliminary study. J Consult Clin Psychol. 1998;66(3):574-8.

34. Butler CC, Rollnick S, Cohen D, Bachmann M, Russel I, Stott N. Motivational consulting versus brief advice for smokers in general practice: a randomized trial. Br J Gen Pract. 1999;49 (445): 611-6.

35. Smith SS, Jorenby DE, Fiore MC, Anderson JE, Mielke MM, Beach KE, et al. Strike while the iron is hot: can stepped-care treatments resurrect relapsing smokers? J Consult Clin Psychol. 2001;69(3): 429-39.

36. Horn K, Fernandes A, Dino G, Massey CJ, Kalsekar I. Adolescent nicotine dependence and smoking cessation outcomes. Addict Behav. 2003;28(4): 769-76.

37. McCambridge J, Strang J. The efficacy of a single-session motivational interviewing in reducing drug consumption and perceptions of drug-related risk and harm among young people: results from a multi-site cluster randomized trial. Addiction. 2004; 99(1):39-52.

38. Stotts AL, DiClemente CC, Dolan-Mullen P. One-to-one: a motivational intervention for resistant pregnant smokers. Addict Behav. 2002;27(2):275-92.

39. Ershoff DH, Quinn VP, Boyd NR, Stern J, Gregory M, Wirstschafter D. The Kaiser Permanente Prenatal Smoking-Cessation Trial: When more isn't better, what is enough? Am J Prev Med. 1999; 17(3):161-8.

\section{ب) Correspondência}

Wilson Vieira Melo

Rua Santa Cecília, 1556, Bairro Santa Cecília

CEP 90420-040, Porto Alegre, RS

E-mail: melo@terapiascognitivas.com.br

Recebido em 28/03/2005.

Aceito em 15/02/2006. 
* O presente trabalho faz parte da Dissertação de Mestrado em Psicologia Clínica do psicólogo Wilson Vieira Melo, intitulada "Estágios motivacionais e tabagismo", defendida no Programa de Pós-Graduação em Psicologia, Faculdade de Psicologia, Pontifícia Universidade Católica do Rio Grande do Sul (PUCRS), no ano de 2005. Este trabalho recebeu incentivo da Coordenação de Aperfeiçoamento de Pessoal de Nível Superior (CAPES). 